\title{
Piotr SORBET
}

Uniwersytet Marii Curie-Skłodowskiej w Lublinie

lepierre@o2.pl

\section{LES MOTS D'ORIGINE PORTUGAISE EN FRANÇAIS}

\section{INTRODUCTION}

La France et le Portugal sont des pays où les langues les plus importantes, du point de vue du nombre des locuteurs, sont d'origine romane. Bien qu'il n'existe pas de voisinage géographique direct entre ces deux pays, au cours des siècles, le français et le portugais se sont influencés mutuellement à maintes reprises. Nous pouvons constater, sans le moindre doute, que le français a été l'adstrat le plus important, s'il s'agit des emprunts, pour l'ensemble du domaine galicien-portugais déjà depuis l'époque médiévale grâce, entre autres, à la lyrique troubadour (Ferreiro 2001 : 312). Soulignons que cette influence ne se limite pas qu'à des mots dispersés mais aussi elle trouve ses reflets dans les calques, la représentation graphique de certains sons, les tournures grammaticales et même dans la morphologie.

Dans le lexique portugais il y a de nombreux mots d'origine française tels que : chefe, chique, chofer, restaurante, etc. Parmi les calques, indiquons à titre d'exemples : estar ao corrente ( $<$ fr. être au courant), pequeno-almoço ( $<$ fr. petit déjeuner), máquina a vapor ( $<$ fr. machine à vapeur) (Walter $1994: 264)$.

En ce qui concerne la graphie, rappelons que le digraphe $c h$ portugais et galicien, utilisé déjà dans le Testamento de Afonso II de 1214 (dans Sancho, chus) est d'origine française (Teyssier 2007 : 22). Il correspondait à cette époque à l'affriquée post-alvéolaire $[\widehat{t} \mathrm{f}]$, devenue en français moderne fricative post-alvéolaire [J] (Walter 1994 : 261). 
En morphologie, il est probable que le suffixe portugais -age (auj. -agem) et le galicien -axe que nous retrouvons dans les formes modernes des mots portugais et galiciens suivants, respectivement: linhagem, mensagem, selvagem et liñaxe, mensaxe, linguaxe soient d'origine française (Walter 1994 : 247, Ferreiro 2001² [1997] : 122-123).

Comme nous venons de l'illustrer, le français a exercé sur le portugais et le galicien une influence sur divers niveaux de la langue. Du point de vue des emprunts, c'est lui qui a contribué le plus à l'accroissement du lexique aussi bien en portugais qu'en galicien, bien que beaucoup de gallicismes dans ces deux langues soient tombés en désuétude (Ferreiro $2001^{2}$ [1997] : 312-315). En revanche, l'influence portugaise (et galicienne) sur le français ne se limite pratiquement qu'au domaine du vocabulaire. Les lusophonismes, bien que du point de vue de leur nombre, ils soient moins importants que le nombre des anglicismes, germanismes, italianismes ou même hispanismes (Sorbet 2014 : 99-101), ne doivent pas être sous-estimés. Il est vrai que la grande partie d'entre eux ne provient pas, en fin de compte, du Portugal mais il ne faut pas oublier que le portugais a joué un rôle d'un important intermédiaire entre le français, voire les langues européennes, avec les langues exotiques parlées dans les régions et les pays où les Portugais avaient établi leurs colonies où ils étaient entrés en contacts commerciaux, culturels et linguistiques avec d'autres peuples.

En effet, le $X V^{e}$ siècle a été marqué par les grandes découvertes. Le Portugal prend Ceuta en 1415, au Maroc, il découvre Porto Santo en 1418, puis il colonise Madère en 1425 et les Açores en 1432 (Cardeira 2006 : 65-66, Teyssier 2007 : 62-79). En plus, entre les années 1488 et 1550, les Portugais, guidés par de grands navigateurs, arrivent successivement en Afrique du Sud au cap de Bonne-Espérance en 1488 (Bartolomeu Dias), aux Indes en 1498 (Vasco de Gama), au Brésil en 1500 (Pedro Álvarez Cabral) et même jusqu'en Chine et au Japon. Ils établissent grâce à leurs découvertes, leurs colonies, entre autres, dans les actuels Cap-Vert, Guinée-Bissau, Sao Tomé-et-Principe, Angola, Mozambique, Timor Oriental et Brésil. Dans ce dernier pays, le portugais a pu s'enraciner définitivement par le moyen du Traité de Tordesillas (signé par Ferdinand II d'Aragon, Isabel I ${ }^{\text {re }}$ de Castille et Jean II de Portugal) de 1494. En vertu de ce traité, on a séparé les domaines d'expansion de la Castille et du Portugal, ce qui a permis l'inclusion du Brésil au monde lusophone et pratiquement tout le reste de l'Amérique Latine au monde hispanophone. 
Les grandes découvertes ont favorisé les contacts linguistiques du portugais avec les langues des peuples des territoires où ils étaient arrivés. Ces contacts ont donné, comme résultat, la possibilité d'emprunter par le français, par le truchement du portugais, à de nombreuses langues exotiques avec lesquelles le français soit n'avait pas de contact direct soit il n'en avait pas établi. Par conséquent, une grande partie des vocables français d'origine portugaise sont des mots qui proviennent, en fin de compte, de l'Amérique du Sud (tupi-guarani), de l'Asie (hindi, marathe, malayalam, tamoul, cinghalais, malais, chinois, japonais) et à moindre échelle de l'Afrique (bantou). N'oublions pas qu'à côté du portugais, l'espagnol, à peu près à la même époque, a joué un rôle étroitement similaire. Pour cette raison, les deux langues ibériques ont transmis, alors, un nombre considérable de mots de diverses provenances.

\section{EMPRUNT LEXICAL}

Jusqu'ici nous avons déjà utilisé le terme d'emprunt, sans préciser le sens sous lequel nous l'utilisons. Nous sommes persuadés que les définitions et théories qui portent sur l'emprunt linguistique ne sont pas toujours convergentes (Sorbet 2014 : 98). Chez les spécialistes, nous pouvons retrouver des opinions selon lesquelles, il peut être identifié avec un élément (Deroy 1956 : 2), un processus (Rey-Debove 1973 : 109-110), une reproduction d'un modèle étranger (Haugen 1950 : 212) ou un mot (Tagliavini 1993 [1973] : 368). Précisons que, même si nous nous appuyons sur les exemples des gallicismes en portugais et en galicien que nous avons évoqués plus haut, nous pouvons constater que l'emprunt n'est pas toujours une unité lexicale et il peut aussi concerner d'autres domaines de la langue que le vocabulaire : la graphie, la morphologie, la syntaxe, etc. Nous soutenons, alors, que l'emprunt lexical est indéniablement un hyponyme de l'emprunt linguistique. Pour cette raison, eu égard au caractère de cette contribution et au type d'emprunts sur lequel nous allons nous concentrer, nous avons décidé, cette fois, d'identifier l'emprunt lexical avec son hyperonyme, c'est-à-dire, l'emprunt linguistique. En plus, dans cette recherche nous allons utiliser le terme de lusophonisme pour nommer n'importe quel mot français dont l'étymon direct est une unité lexicale du portugais (européen, brésilien, etc.). 


\section{CORPUS}

Afin de réaliser notre travail nous avons réuni quelques sources de quatre caractères fondamentaux. En premier lieu, nous avons consulté quatre dictionnaires étymologiques : le Nouveau Dictionnaire étymologique et historique (DED), le Dictionnaire étymologique et historique de la langue française (DEB), le Dictionnaire des mots d'origine étrangère (DMEH) et le Dictionnaire historique de la langue française (GREH). En deuxième lieu, nous avons pris en considération deux publications de la série de dictionnaires généraux des éditions Robert, c'est-à-dire, Le Petit Robert $2001\left(R E_{2001}\right)$, le Petit Robert $2014\left(R E_{2014}\right)$ et afin de réaliser une observation particulière que nous allons annoncer infra le Larousse pratique : Dictionnaire $d u$ français au quotidien $(L P)$. En dernier lieu, nous nous sommes basés sur quelques travaux linguistiques. Ceux qui nous ont servi le plus sont les suivants : Les mots étrangers (Guiraud 1971) et L'aventure des mots français venus d'ailleurs (LMW). Malgré la diversité de sources consultées, quelquefois, nous avons été obligés d'admettre que l'établissement avec sûreté de l'origine de certains mots est, soit impossible, soit reste encore à examiner plus profondément. Dans ces cas, nous avons décidé de conférer à ces emprunts le signe (?) pour montrer que la marque qui indique leur origine ne manque pas d'un certain degré d'incertitude.

\section{ANALYSE QUANTITATIVE}

Nous avons déjà affirmé qu'identifier l'origine de certains mots est pratiquement impossible. Ceci est dû à deux raisons principales.

En premier lieu, les dictionnaires fournissent des informations contradictoires ou incomplètes parce que tantôt ils prennent en considération l'étymologie directe, tantôt l'étymologie indirecte et, parfois même, ils mélangent les deux points de vues.

En deuxième lieu, le portugais a été longtemps concurrencé par l'espagnol en ce qui concerne l'importation des mots d'origine exotique, mais avant tout, de l'Amérique Latine. En le sachant et ayant en mémoire que les deux langues sont étroitement apparentées (ce sont des langues romanes du type ibérique), souvent les équivalents lexicaux des deux idiomes en question sont semblables ce qui, parfois, fait obstacle à l'indication de la voie par laquelle un mot est arrivé en français, c'est-à-dire, la voie portugaise ou la voie espagnole. 
Il y a un certain temps, à l'occasion d'une autre contribution, nous avons déjà réalisé un travail de comptage des mots d'origine ibérique dans la langue française (Sorbet 2014). Il s'est avéré que, du point de vue du nombre des vocables comptés dans le $R E_{2001}$ et le $R E_{2014}$, l'idiome ibérique qui a contribué le plus au français c'est l'espagnol dépassant presque quadruplement le portugais (Sorbet 2014 : 100).

La période où le français a emprunté au portugais ont été les siècles dans le cadre de l'époque des Grandes découvertes mais surtout au cours du XVI ${ }^{\mathrm{e}}$ et du XVII ${ }^{\mathrm{e}}$ siècles (Sorbet $2014: 101$ ).

\begin{tabular}{|c|c|c|c|c|c|c|c|c|}
\cline { 2 - 9 } \multicolumn{1}{c|}{} & $\mathrm{XV}^{\mathrm{e}}$ & $\mathrm{XVI}^{\mathrm{e}}$ & $\mathrm{XVII}^{\mathrm{e}}$ & $\mathrm{XVIII}^{\mathrm{e}}$ & $\mathrm{XIX}^{\mathrm{e}}$ & $\mathrm{XX}$ & Total & \\
\hline $\mathrm{RE}_{2001}$ & 3 & 38 & $\mathbf{3 9}$ & 16 & 21 & 11 & $\mathbf{1 2 8}$ & \multirow{2}{*}{ lusophonismes } \\
\hline $\mathrm{RE}_{2014}$ & 3 & 35 & $\mathbf{5 1}$ & 16 & 22 & 15 & $\mathbf{1 4 2}$ & \\
\hline
\end{tabular}

Bien entendu, ces données doivent être traitées comme approximatives et non pas définitives. Constatons que le $R E_{2014}$ enregistre plus de lusophonismes que le $R E_{2001}$. Ceci est dû, d'une part, au fait que le premier répertoire de nouveaux mots, et d'autre part, que les informations étymologiques y sont, parfois, révisées en faveur ou en défaveur du portugais. Analysons quelques entrées.

Le $R E_{2014}$ recueille une série de mots qui ne figurent pas dans le $R E_{2001}$, par exemple caïpirinha, matabiche ou saudade. L'intermédiaire du portugais du mot hindi bandana (?), indiqué dans le $R E_{2001}$, est remis en question dans le $R E_{2014}$ où on signale comme intermédiaire l'anglais américain. Pour ce qui est du mot paillote 'cabane, hutte de paille' (?) le $R E_{2001} l^{\prime}$ indique comme un dérivé de paille, mais le $R E_{2014}$ ne réfute pas l'origine portugaise. Par ailleurs, le GREH suggère, pour cette forme, un emprunt au portugais palhota.

Il arrive que l'origine d'un mot, à cause de la ressemblance des formes portugaises et espagnoles, ce que nous avons déjà avancé, reste une question discutable. Le substantif albinos (?), selon le $R E_{2001}$, est un lusophonisme, en revanche, le $R E_{2014}$ lui confie la marque d'hispanisme. Parallèlement, on attribue aux vocables tels que : mangouste (?) 'petit mammifère' ou nègre (?), dans les deux éditions du dictionnaire, une provenance soit portugaise soit espagnole ${ }^{1}$.

1 Le $L P$, le $D E B$ et le $L M W$ lui confèrent la marque d'hispanisme. Nous aussi nous penchons, plutôt, vers l'origine espagnole. 
En plus, la double voie par laquelle les mots arrivaient à la langue française ont permis que le portugais et l'espagnol ont enrichi parallèlement le vocabulaire français afin de désigner un seul objet, un seul concept ou une seule notion. Par exemple, la forme espagnole puma, venant du quechua, attestée en français depuis le XVII ${ }^{\mathrm{e}}$ siècle, et la forme couguar, provenant du tupi mais par le truchement du portugais qui est attestée en français depuis le XVIII ${ }^{\mathrm{e}}$ siècle, désignent le même animal. Le premier d'entre eux est, alors, un hispanisme, et le deuxième un lusophonisme. Similairement, les deux substantifs hacienda et fazenda font appel à une 'grande exploitation agricole', mais le deuxième d'entre eux, se limite à la description de la réalité brésilienne et le premier désigne une exploitation agricole en Amérique du Sud.

Parmi les lusophonismes il est nécessaire de distinguer les emprunts des dérivés. Ces derniers ne sont pas de vrais emprunts, mais leur existence démontre leur adaptation à la langue française. Indiquons à titre d'exemples : port. acaju $>$ fr. acajou $\rightarrow$ cajou ; port. mandarim $>$ fr. mandarin $\rightarrow$ mandarinat; port. sagu $>$ fr. sagou $\rightarrow$ sagoutier; port. selva $>$ fr. selve $\rightarrow$ selvatique, etc.

Il est surprenant qu'il y ait des mots qui sont arrivés à la langue française par le truchement du portugais, souvent le brésilien, mais qui ne sont pas enregistrés dans les sources consultées $\left(R E_{2001}, R E_{2014}, D E D\right.$, $D E B, D M E H, G R E H, T L F)$, par exemple, capoeira (et son dérivé capoeiriste) qui provient, en fin de compte, du guarani et qui est catalogué dans le $L P$. D'ailleurs, il serait aussi envisageable de répertorier encore d'autres termes qu'on y utilise tels que : roda 'cercle formé par les capoeiristes', batizado 'passage de grade en capoeira', ginga 'type de mouvement en capoeira', berimbau 'type d'instrument de musique brésilien' méconnus dans les : $R E_{2001}, R E_{2014}, D E D, D E B, D M E H, G R E H$, TLF et $L P$.

\section{ANALYSE QUALITATIVE}

Les observations quantitatives achevées, maintenant, nous pouvons passer à l'analyse qualitative. Soulignons qu'en raison de la limite d'espace de cette contribution, elle se concentrera uniquement sur les aspects les plus importants selon notre perspective.

En ce qui concerne les classes grammaticales, le français a emprunté au portugais presque exclusivement des substantifs. Les adjectifs sont 
définitivement peu nombreux, et en plus, souvent ils peuvent être utilisés comme des substantifs (métis, nègre (?)). Apparemment, le seul verbe français qui peut être d'origine portugaise est le vocable sombrer (?), bien qu'il puisse venir également de l'espagnol ou même du catalan $\left(R E_{2014}\right)$.

L'adaptation orthographique des lusophonismes français, en général, ne pose pas de grands problèmes. Il existe, néanmoins, certains mots qui présentent plus d'une seule graphie : caldeira/caldera, copra/coprah, couguar/cougouar, jambose/jamerose, raja/radja, teck/tek, etc. On peut relever le substantif sertão 'zone semi-aride du Brésil où l'on pratique l'élevage extensif' qui maintient l'orthographe originale (le tilde sur le $a$ est notamment voyant). Il est intéressant qu'il y a des lusophonismes qui varient en orthographe selon le dictionnaire consulté, par exemple, le $R E_{2001}$ indique la graphie bossa-nova, en revanche, dans le $R E_{2014}$ déjà à côté de celle-ci nous retrouvons une deuxième graphie sans le trait d'union bossanova. Ceci prouve que ce mot, peu à peu, s'adapte à l'orthographe française.

Nous constatons certaines hésitations dans la prononciation de quelques mots, ce qui permet d'enregistrer deux ou trois prononciations différentes : cruzado [kruzado ; krusado], escudo [cskydo ; عskudo], jambose [3ãboz] / jamerose [заmRoz], radja [Radza] / rajah [ва(d)за], saudade [sodad; saudad(e)].

À cause des différences phonologiques entre le français et le portugais ainsi que les langues auxquelles remontent, en fin de compte, les mots, certains lusophonismes manifestent des sons étrangers : matchiche [mat $\mathrm{fi}$ ]], radja [Rаđ̧̆a].

Pour ce qui est des catégories grammaticales des emprunts, les substantifs gardent communément le genre de leurs équivalents portugais : port. fado $(\mathrm{m})$, caravela $(\mathrm{f})>$ fr. fado $(\mathrm{m})$, caravelle $(\mathrm{f})$. Néanmoins, il y a certaines exceptions : port. jambo $(\mathrm{m})>$ fr. jambose/jamerose (f), port. piranha $(\mathrm{f})$ $>$ fr. piranha $(\mathrm{m})$, port. zebra (f) > fr. zèbre $(\mathrm{m})$.

Dans les lusophonismes, le nombre grammatical est pratiquement toujours maintenu. Cependant, nous avons retenu quelques cas particuliers. Dans la forme du vocable français albinos (?) nous retrouvons la marque du pluriel -s qui n'est pas ressentie comme telle en français. C'est, alors, un exemple de la dépluralisation de l'étymon portugais ou espagnol (cf. pol. Eskimos 'Esquimau', keks 'cake'). Un autre cas qui mérite un bref commentaire est celui de achards. D'après le $R E_{2001}$, le $R E_{2014}$ et le $L P$ c'est un mot masculin pluriel, donc, un plurale tantum, en revanche, le GREH 
et le TLF indiquent également les formes suivantes : achar/achard pour le singulier et achars/achards pour le pluriel. Ceci peut prouver ce que nous avons déjà annoncé, c'est-à-dire, que les différentes sources, parfois, présentent des informations contradictoires.

S'il s'agit des lusophonismes français il est intéressent de remarquer que parmi eux il arrive qu'un mot qui provient d'une des langues classiques (latin ou grec) forme, avec des mots français hérités du latin, des doublets qui remontent, en fin de compte, au latin :

lat. campus $>$ fr. champ,

lat. campus $>$ port. campo $>$ fr. campo;

lat. scutum $>$ fr. écu,

lat. scutum > port. escudo > fr. escudo ;

lat. niger $>$ fr. noir,

lat. niger $>$ esp., port. negro $>$ fr. nègre ;

lat. facticius $>$ fr. factice,

lat. facticius $>$ port. feitiço $>$ fr. fétiche ;

Dans le dernier cas, il est nécessaire d'observer qu'il s'agit d'un exemple d'un emprunt aller-retour, c'est-à-dire, port. feitiço > fr. fétiche $>$ port. fetiche. Tout comme les substantifs paella et olla-podrida pour ce qui est des emprunts allers-retours dans les contacts linguistiques franco-espagnols (Sorbet 2014 : 103).

Au niveau de l'analyse sémantique, sans le moindre doute, les lusophonismes gardent une relation sémantique avec leurs étymons portugais, bien que les premiers, parfois, expérimentent une certaine limitation dans le sens ou dans l'usage. Examinons quelques exemples:

Le substantif français fado 'type de chant portugais' (< port. fado) n'a pas la signification de 'sort ou destin' de son étymon. Similairement, escudo fait seulement appel à 'l'unité monétaire portugaise', cependant, en portugais il peut aussi signifier, entre autres, 'bouclier, écu'. Le lusophonisme manuélin ( $<$ port. manuelino) ne peut pas être utilisé comme substantif comme son équivalent formel portugais.

Une grande partie des lusophonismes peut être groupée en plusieurs realia de différents pays :

a) Les realia d'origine portugaise.

Escudo, fado, manuélin, porto, saudade.

b) Les realia d'origine brésilienne.

Bossa-nova, campo, cruzado, cruzeiro, favela, fazenda, lambada, matchiche, samba, selve, seringuero, sertão. 
c) Les realia d'origine hindi.

Bayadère, cipaye, nabab, paria, raja, roupie.

d) Les realia de l'extrême-orient (la Chine et le Japon).

Bonze, cangue, mandarin, pagode, tael.

En plus, nous sommes convaincus qu'il faut distinguer, au moins d'une manière arbitraire, certains champs sémantiques parmi lesquels les plus importants, du point de vue du nombre, sont les suivants :

a) Gastronomie.

Achards, caïprinha, madère (avec lexicalisation), marmelade, porto (avec lexicalisation).

b) Botanique (plantes, fruits, légumes, etc.).

Ananas (?), aréquier, bambou, banane, banian, bétel, copra(h), datura, igname, ipéca, jambose/jamerose, lilas, litchi, longane, mangoustan, mangue, négondo, palmiste, pastèque, pétun, piassava, tapioca.

c) Zoologie.

Cachalot, coati, cobaye, cobra, couguar/cougouar, jaguar, macaque, mangouste, pintade, piranha, sagouin, saïmiri, sarigue, serval, tamandua, zèbre.

d) Arts au sens large.

Baroque, bossa-nova, fado, griot (?), lambada, manuélin, matchiche, samba.

e) Religion et croyances.

Autodafé, bayadère, bonze, candomblé, fétiche, marabout, pagode.

f) Géographie.

Caldeira, campo, selve, sertão.

g) Navigation et termes maritimes.

Argousin, balise, caravelle, écubier (?), jangada, jonque (?), métis, mousson, prao, typhon (?), vigie.

h) La société et son fonctionnement.

Caste, cipaye, cruzeiro, cruzado, favela, fazenda, nabab, paria, radja, roupie, tael.

Il y a aussi des termes que nous n'avons pas pu classer : alastrim, aniline, bandana, cachou, calambac, cangue, carbonado, cornac, lascar, nègre (?), palanquin, sagou, seringuero, varague, véranda.

\section{EN MANIÈRE DE CONCLUSION}

Les lusophonismes que nous avons présentés ne constituent pas l'ensemble de l'influence de la langue portugaise sur le français. Nous 
n'avons par abordé le problème de l'influence limitée diatopiquement (pays africains, Guyane), bien que celle-ci existe. Pour le prouver, il suffit de mentionner : matabiche (< port. mata-bicho) qui signifie en Afrique noire 'pourboire, petit cadeau, pot-de-vin' $\left(R E_{2014}\right)$.

Nous n'avons pas non plus évoqué, dans cet article, les mots que nous avions repérés dans des sources spécialisées, par exemple, de la gastronomie : bragance 'garniture pour tournedos', caldeirada 'type de bouillon portugais', etc. En pensant qu'inclure ce type de vocables agrandirait considérablement notre travail. En plus, nous sommes convaincus que ces lusophonismes mériteront notre attention dans une autre contribution.

\section{RÉFÉRENCES}

Baumgartner E., Ménard P., 1996, Dictionnaire étymologique et historique de la langue française, Paris. (DEB)

Cardeira E., 2006, O ossencial sobre a história do português, Lisboa.

Dauzat A., Dubois J., Mitterand H., 1971, Nouveau Dictionnaire étymologique et historique, Paris. (DED)

Deroy L., 1956, L'emprunt linguistique, Paris.

Ferreiro M., 2001² [1997], Gramática histórica galega II. Lexicoloxía, Santiago de Compostela.

Guiraud P., 1971, Les mots étrangers, Paris.

Haugen E., 1950, «The analysis of linguistic borrowing », Language 26, 210-231.

Delacherie-Henry S., Nief C., Vandevoorde V. avec la collab. de Morel D., 2003, Larousse pratique : dictionnaire du français au quotidien, Paris. (LP)

Le Petit Robert 2001 [cédérom], Paris. $\left(\mathrm{RE}_{2001}\right)$

Le Petit Robert 2014 [cédérom], Paris. $\left(\mathrm{RE}_{2014}\right)$

Rey A., $2006^{3}$, Dictionnaire historique de la langue française, Paris. (GREH)

Sorbet P., 2014, "La gastronomie ibérique dans la langue française», Revue d'Études Française 19, 97-105.

Tagliavini C., 1993² [1973], Orígenes de las lenguas neolatinas: introducción a la filología romance, México.

Teyssier P., 2007, História da língua portuguesa, São Paulo.

Trésor de la langue française, en ligne [http://www.tresor-de-la-langue-francaiseinformatise.fr/]. (TLF)

Walter H., 1994, L'aventure des langues en Occident, Paris.

Walter H., 1997, L'aventure des mots français venus d'ailleurs, Paris. (LMW)

Walter H., Walter G., 2009, Dictionnaire des mots d'origine étrangère, Paris. (DMEH) 


\section{LES MOTS D'ORIGINE PORTUGAISE EN FRANÇAIS}

\section{Résumé}

Les relations linguistiques entre la France et les pays lusophones, surtout le Portugal, ont une longue tradition. Sans le moindre doute, ces relations ont amené les langues ibériques à emprunter plus au français qu'inversement. Cependant, le portugais, au cours des siècles, a aussi fourni à la langue française de nombreux mots. Bien entendu, pas tous ces mots, proviennent, en fin de compte, de la Péninsule Ibérique mais le Portugal a pu joué un rôle d'intermédiaire entre le français et, d'une part, le portugais dans ses variantes hors-européennes, et d'autre part, certaines langues américaines, africaines et asiatiques. Ainsi nous voulons, dans cette contribution, nous occuper des vocables que nous pouvons dénommer grosso modo comme des lusophonismes.

Mots-clés : emprunts lexicaux, lusophonismes, étymologie, contacts linguistiques

\section{THE WORDS OF PORTUGUESE ORIGIN IN FRENCH}

\section{Summary}

The language relations between France and lusophone countries, especially Portugal, have a long tradition. Without a doubt, these relations have led Iberian languages to borrow more from French than vice versa. However, Portuguese has also contributed many words to French over centuries. Certainly, not all of these words come ultimately from the Iberian Peninsula but Portugal has played the role of intermediary between French and, Portuguese in its non-European variants as well as certain American, African and Asian languages. Thus, the main focus of this paper is the study of words which can be roughly classified as lusophonisms.

Key words: loanwords, lusophonisms, etymology, language contacts 